\title{
Study of the Characteristics and Computation Analysis Results of Electromechanical Systems Models
}

\author{
Berdai Abdelmajid, Abdelhadi El Moudden \\ National School of Electricity and Mechanics, ENSEM \\ University Hassan II Ain Chock, Casablanca, Morocco. \\ E-mail: a.berdai@gmail.com; a.elmoudden@yahoo.fr
}

\author{
Chornyi O.P. \\ Kremenchuk Mykhailo Ostrohradskyi National University, Kremenchuk Ukraine \\ E-mail:apch@kdu.edu.ua;
}

\begin{abstract}
Today, simulation of electrical drives with asynchronous motors based on systems of differential equations is regarded as one of the principal means of their research study. The difficulty of the simulation is determined by the need for accuracy of the results obtained and the complexity of the mathematical model's differential equations. In this article, we present a study of the particularities of the simulation of electrical drives systems with asynchronous motors. We have studied models composed of three-phase and orthogonal coordinates systems and we have shown that qualitative and quantitative differences exist in the process of changing the angular speed of the rotor and electromagnetic torque. The result obtained is above all influenced by the non-linear character of the load opposing a fan-type or "dry friction”-type resistant torque. For dual-earthed electromagnetic actuation with the moments of the resistant torques indicated, integration of differential equation systems was carried out with various digital methods used in professional mathematical software for simulation.
\end{abstract}

Index Terms - electromechanical system model, differential equations, discontinuous functions

\section{Introduction.}

Simulation and research into electromechanical systems (EMS) and apparatus on computers is a firmly established research practice [1,2], sometimes even replacing theoretical research or analysis based on analytically obtained expressions. Researchers are gradually moving away from simplified mathematical models, and considering models based on differential or integral equations taking account of the electrical circuit as a whole and the various forms of magnetic or electromagnetic interaction. Understanding of the physical nature of equipment and the possibilities of computer programs for simulation is therefore limited. But with increasing complexity of the model, a new problem appears - to effectively and efficiently correct systems of differential equations (SDE) resolution we have to set the nature of the computational problem and choose the best method.

\section{Analysis of previous studies.}

With the possibilities of expansion of computer equipment, the study of electromechanical systems is carried out by mathematical and object-oriented software packages (tab.1).

As a rule, Simulation Software Packages contain a 
set of methods enabling problems of various types to be solved [3]. Real electromagnetic and electromechanical processes in the EMS, as inculcated, are described by rigid systems of differential equations (SDE). However, we often meet enough fast oscillating systems which describe the high-frequency fluctuations and locally unstable system, in the choice of zones with divergent processes alternating with more stable zones.

With integration of stiff systems, it is necessary to provide fast attenuation of rigid components. To do this, we can use implicit A- or L stable methods [4, 6]. The character of the problem can be changed during the resolution process or when passing from one variable to another. For solving such problems we have to use effective adaptive methods [7] whose settlement formulas are adjusted on systems that integrate.

Tab.1 Some mathematical and object-oriented software packages to study electromecanical systems EMS.

\begin{tabular}{|l|l|}
\hline $\begin{array}{l}\text { Mathematica, } \\
\text { MatLab, }\end{array}$ & $\begin{array}{l}\text { Interactive system for performing } \\
\text { engineering and scientific } \\
\text { calculations }\end{array}$ \\
\hline Mathcad & $\begin{array}{l}\text { Environment for execution on a } \\
\text { computer of a variety of } \\
\text { mathematical and engineering } \\
\text { calculations }\end{array}$ \\
\hline Model Vision & $\begin{array}{l}\text { Computer lab to simulate and } \\
\text { study complex dynamic systems }\end{array}$ \\
\hline Studium & $\begin{array}{l}\text { Package design and simulation of } \\
\text { electrical circuits }\end{array}$ \\
\hline WorkBench & $\begin{array}{l}\text { Universal package for the } \\
\text { simulation of electric machines } \\
\text { and semiconductor converters }\end{array}$ \\
\hline VisSim & $\begin{array}{l}\text { Software package for the } \\
\text { simulation of complex electrical } \\
\text { systems and their analysis. }\end{array}$ \\
\hline
\end{tabular}

Difficulties appear during the integration of systems with discontinuous right hand sides [9]. For correct resolution of such problems, it is necessary to reduce the integration step in the vicinity of break points, which adds further requirements to the procedure of controlling the value of the step; besides this, there are possible even pseudo hang solution due to the significant reduction step.

New mathematical software uses a series of methods for numerical solution of differential equation systems. For example, in Matlab-simulink seven methods are implemented with automatic choice of integration fixed steps :

- Methods with constant step integration: ode5 - is the fixed-step version of ode45 (Dormand-Prince formula); ode4 is RK4, the fourth-order Runge-Kutta formula; ode3 - is the fixed-step version of ode23, the Bogacki-Shampine formula; ode2 - is Heun's method, also known as the improved Euler formula; ode1 - is Euler's method.

- Methods with variable step integration: ode45 - is based on an explicit Runge-Kutta $(4,5)$ formula, the Dormand-Prince pair, for the resolution of non-stiff differential equation systems; ode23 - is an implementation of an explicit Runge-Kutta $(2,3)$ pair of Bogacki and Shampine, for the resolution of non-stiff and moderately stiff differential equation systems; ode113 - is a variable order Adams-Bashforth-Moulton PECE solver. It may be more efficient than ode45 at stringent tolerances and when the ODE file function is particularly expensive to evaluate. ode113 is a multistep solver - it normally needs the solutions at several preceding time points to compute the current solution; ode15s - is a variable order solver based on the numerical differentiation formulas (NDFs). Optionally, it uses the backward differentiation formulas (BDFs, also known as Gear's method) that are usually less efficient. Like ode113, ode15s is a multistep solver. Try ode $15 \mathrm{~s}$ when ode45 fails, or is very inefficient, and you suspect that the problem is stiff, or when solving a differential-algebraic problem; ode $23 \mathrm{~s}$ - is based on a 
modified Rosenbrock formula of order 2. Because it is a one-step solver, it may be more efficient than ode $15 \mathrm{~s}$ at crude tolerances. It can solve some kinds of stiff problems for which ode15s is not effective; ode23t is an implementation of the trapezoidal rule using a "free" interpolant. Use this solver if the problem is only moderately stiff and you need a solution without numerical damping. ode23t can solve DAEs; ode23tb is an implementation of TR-BDF2, an implicit Runge-Kutta formula with a first stage that is a trapezoidal rule step and a second stage that is a backward differentiation formula of order two. By construction, the same iteration matrix is used in evaluating both stages. Like ode23s, this solver may be more efficient than ode $15 \mathrm{~s}$ at crude tolerances. It's used for the resolution of stiff differential equation systems.

This group of methods enables effective resolution of a number of tasks, but, at the same time, the problem of choosing the corresponding method and its parameters became apparent. As a general rule, there are few recommendations put forward in the reference literature [13], and choice of method and its parameters is entirely determined by the researcher's level of knowledge and qualification.

\section{Objective of the work.}

Research particularities of calculation models of the electromechanical systems

\section{Material and research results}

For the resolution of rigid and non-rigid problems, concrete recommendations are given in [2 - 4].

We must take into account that often on the right side of differential equations systems DES of electromecanical systems EMS, models include the output voltage power thyristor converter or power transistor converter; nonlinear characteristics such as a "Dry friction" or "Backlash".etc. These signals are characterized by first-order discontinuities which suggest that the DES of EMS models are discontinuous alignment.

In the first phase of the work, the problems of modelling of Van der Pol oscillator with discontinuous function were studied.

$\frac{d^{2} y}{d t^{2}}=\operatorname{sign}(1-|y|) \frac{d y}{d t}-y$

The mechanical part of the electric drive system with torque -type "dry friction" was also studied [10].

As a study object, we consider a mathematical model of a two-mass system with reactive torque resistance mechanism and with a gap in the transmission. The system of equations model is of type (2).

$J_{1} \frac{d \omega_{1}}{d t} M_{d}-M_{y}$

$J_{2} \frac{d \omega_{2}}{d t} M_{y}-M_{c} \operatorname{sign}\left(\omega_{2}\right)$;

$\frac{d \Delta \varphi}{d t} \omega_{1}-\omega_{2}$

$M_{y}=\left\{\begin{array}{l}0 \quad n p u|\Delta \varphi| \leq \frac{\delta}{2} ; \\ c\left(\Delta \varphi-\frac{\delta}{2} \operatorname{sign}(\Delta \varphi)\right) n p u \quad|\Delta \varphi|>\frac{\delta}{2},\end{array}\right.$

where $-J_{1}, J_{2}, \omega_{1}, \omega_{2}$ the moments of inertia and rotational speed, respectively, the $1^{\text {st }}$ and $2^{\text {nd }}$ of mass;

$M_{d}, M_{y}, M_{c^{-}}$Torques respectively motor, elastic and resistant, c - stiffness coefficient,

$\Delta \varphi$ - torsion angle of the shaft, $2 \delta$ - gap , rad.

the study of system (2) is performed for: $J_{1}=0,1$ $J_{2}=0,5$ кгм ${ }^{2}, M_{d}=40 \quad M_{c}=5 \mathrm{Hм}, c=5000$.

Figures 2 and 4

show the study results of integration of the systems using the methods with constant step and variable step.

It should be noted that the calculation of systems taking the nonlinearities into account, could only be performed by disabling previously option «Zero crossing control»- position with «Disable All». But in this case, by taking into account precision of 8.10 , methods with variable step of integration has not provided the 
calculation - in fact, they were stuck. The only exception was ode15s and ode23t. Thus, the latter obviously performed the calculation incorrectly. A decrease in Precision down to $10^{-6}$ allows for calculation with whatever method.

Thereafter, this difference is clearly noticeable in the results obtained.

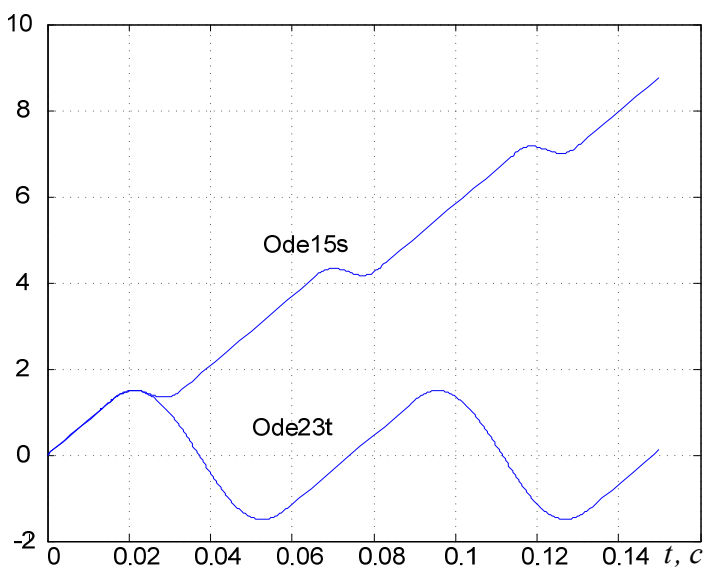

Figure 2. The calculation of (2) with the reactive torque at precision $10^{-8}$ using methods with variable step integration.

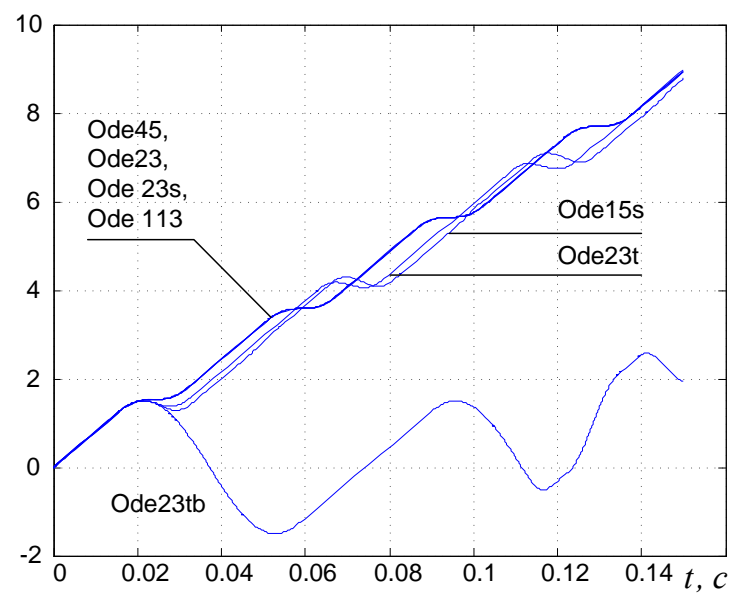

Figure 3. The calculation of (2) with the reactive torque at precision $10^{-6}$ using methods with variable step integration.

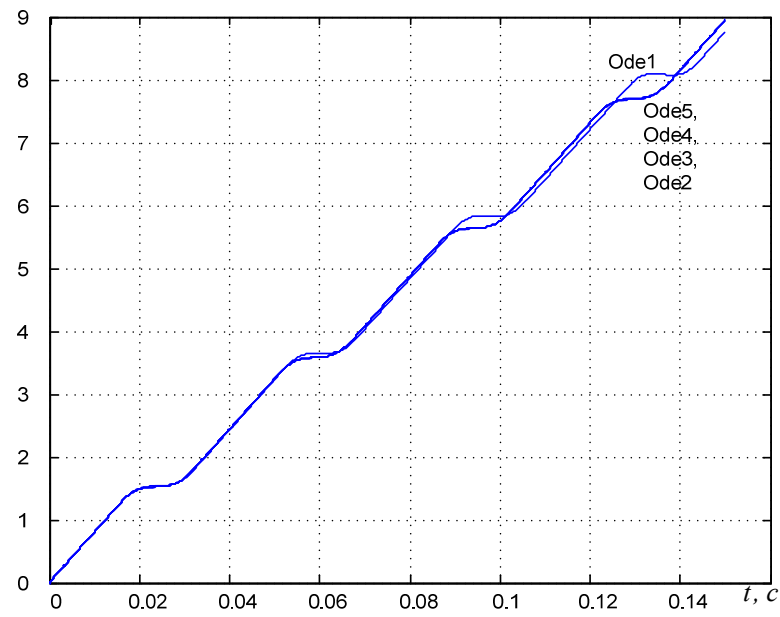

Figure 4. The calculation of system (2) with a reactive nature of the moment with an accuracy of $10^{-4}$ using methods with constant step integration

As a study object, we consider a mathematical model of asynchronous motor AC motor, which drives a ventilator.

Note that the system with electrical machines is nonlinear. Nonlinearity is mainly determined by two factors

- Dependence system parameters (resistance and inductance, coefficients of magnetization, gain of the regulators, etc.) of mode parameters.

- Nonlinear coupling mode parameters of the system between them.

Nonlinearity of the system parameters is usually not considered, except for special cases.

As a rule, Nonlinear coupling parameters of the system are taken into account.

For simplicity's sake, we consider that the orthogonal model of AC motor in the system coordinate $\alpha, \beta$ and $u, v$ : 


$$
\begin{aligned}
& \left\{\begin{array}{l}
\frac{d \psi_{\alpha s}}{d t}=U_{\alpha s}-A R_{s}\left(\psi_{\alpha s} L_{r}^{\prime}-\psi_{\alpha r} L_{\mu}\right) \\
\frac{d \psi_{\beta s}}{d t}=-U_{\beta s}-A R_{s}\left(\psi_{\beta s} L_{r}^{\prime}-\psi_{\beta r} L_{\mu}\right) \\
\frac{d \psi_{\alpha r}}{d t}=-A R_{r}^{\prime}\left(\psi_{\alpha r} L_{s}-\psi_{\alpha s} L_{\mu}\right)+\psi_{\beta r} \omega \\
\frac{d \psi_{\beta r}}{d t}=-A R_{r}^{\prime}\left(\psi_{\beta r} L_{s}-\psi_{\beta s} L_{\mu}\right)+\psi_{\alpha r} \omega \\
M=\frac{3}{2} p L_{\mu} A\left(\psi_{\alpha s} \psi_{\beta r}-\psi_{\beta s} \psi_{\alpha r}\right) \\
\frac{d \omega}{d t}=\frac{1}{J}\left[M-M_{c}\left(\frac{\omega}{\omega_{0}}\right)^{2}\right]
\end{array}\right. \\
& \text { and } \\
& \left\{\begin{array}{l}
\frac{d \psi_{1 u}}{d t}=U_{l u}-\frac{R_{s} \Psi_{l u}}{L_{r}^{\prime} \sigma}+\frac{R_{s} L_{\mu} \Psi_{2 u}}{L_{r}^{\prime} L_{s} \sigma}+\omega_{0} \Psi_{l v} ; \\
\frac{d \psi_{1 v}}{d t}=U_{l v}-\frac{R_{s} \Psi_{1 v}}{L_{r}^{\prime} \sigma}+\frac{R_{s} L_{\mu} \Psi_{2 v}}{L_{r}^{\prime} L_{s} \sigma}-\omega_{0} \Psi_{l u} ; \\
\frac{d \Psi_{2 u}}{d t}=-\frac{R_{r} \Psi_{2 u}}{L_{s} \sigma}+\frac{R_{r} L_{\mu} \Psi_{l u}}{L_{r}^{\prime} L_{s} \sigma}+\left(\omega_{0}-p \omega\right) \Psi_{2 v} ;
\end{array} ;\right. \\
& \left\{\frac{d \psi_{2 v}}{d t}=-\frac{R_{r} \Psi_{2 v}}{L_{s} \sigma}+\frac{R_{r} L_{\mu} \Psi_{1 v}}{L_{r}^{\prime} L_{s} \sigma}+\left(\omega_{0}-p \omega\right) \Psi_{2 u}\right. \text {; } \\
& M=\frac{3}{2} p \frac{L_{\mu}}{L_{r}^{\prime} L_{s} \sigma}\left(\psi_{1 v} \psi_{2 u}-\psi_{1 u} \psi_{2 v}\right) \text {; } \\
& \frac{d \omega}{d t}=\frac{1}{J}\left[M-M_{c}\left(\frac{\omega}{\omega_{0}}\right)^{2}\right] \text {. }
\end{aligned}
$$

Orthogonal models (3) and (4) were obtained by algebraic and trigonometric transformations of the three-phase model [11] and are linear in parameters but nonlinear in liaison. And they also contain a nonlinear function of speed.

Fig. 5 - 8 show the results of studies with the simulation of systems (3) and (4) as compared with the simulation in the three-phase system.

The integration was performed using the Adams-Beshforts-Moulton multi-step method (ode113) (method for prediction and correction of the variable order) for solving non-rigid SDE, which is effective at high precision [2].

During the simulation we considered regimes starting AC Motor (tab.1), unloaded and under load.
Table 2. Parameters of AC Motor taken in the simulation.

\begin{tabular}{|l|c|}
\hline \multicolumn{1}{|c|}{ Parameter } & Value \\
\hline nominal power, $\mathrm{kW}$ & 4 \\
\hline Nominal voltage, $\mathrm{V}$ & 380 \\
\hline nominal rotational speed, rad / s & 151,2 \\
\hline Nominal torque, Nm & 26,5 \\
\hline Stator resistance, Ohm & 1,66 \\
\hline Rotor resistance, Ohm & 1,28 \\
\hline Stator Leakage inductance ohms & 1,95 \\
\hline rotor Leakage inductance Ohm & 3,36 \\
\hline inductance of magnetic circuit, $\mathrm{H}$ & 59,38 \\
\hline Moment of inertia, kgm ${ }^{2}$ & 0,108 \\
\hline
\end{tabular}

In Fig. 5 - 8, curve 1 - corresponds to the model in three-phase system of coordinates, curve 2 - to that in the coordinates $\alpha, \beta$, curve $3-$ to that in the coordinates $u, v$.
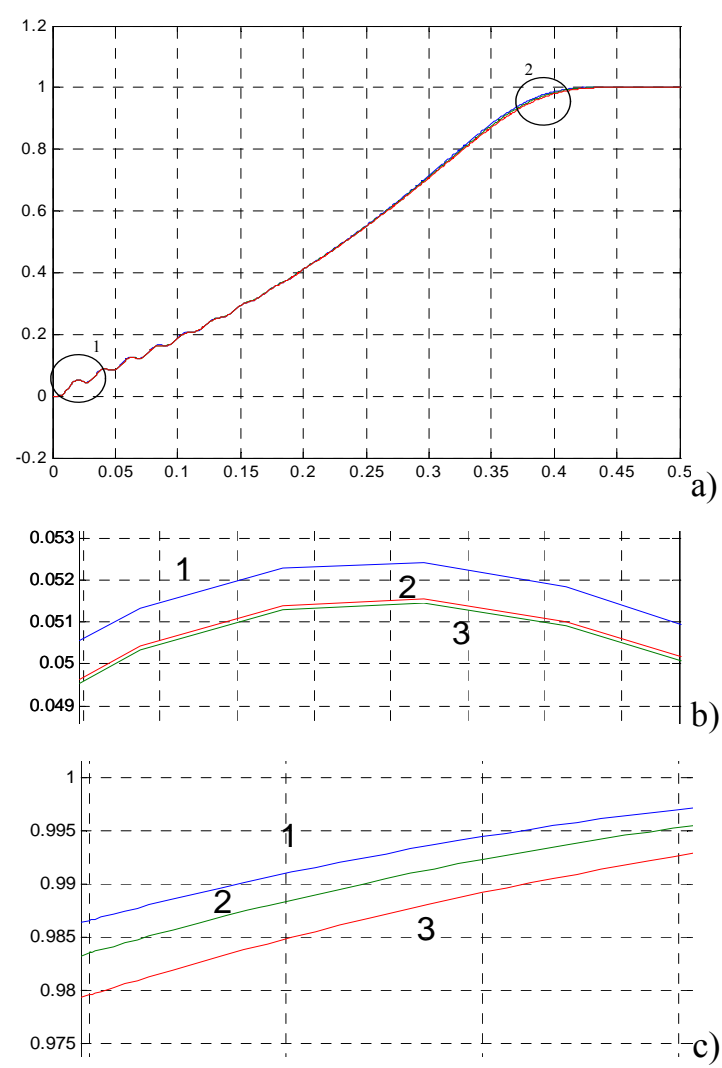

Figure 5.Speed rotation curve as a function of time of AC motor at loadless starting : a) - general view, b) - the first fragment, first oscillation, c) second fragment, access to the stationary regime 

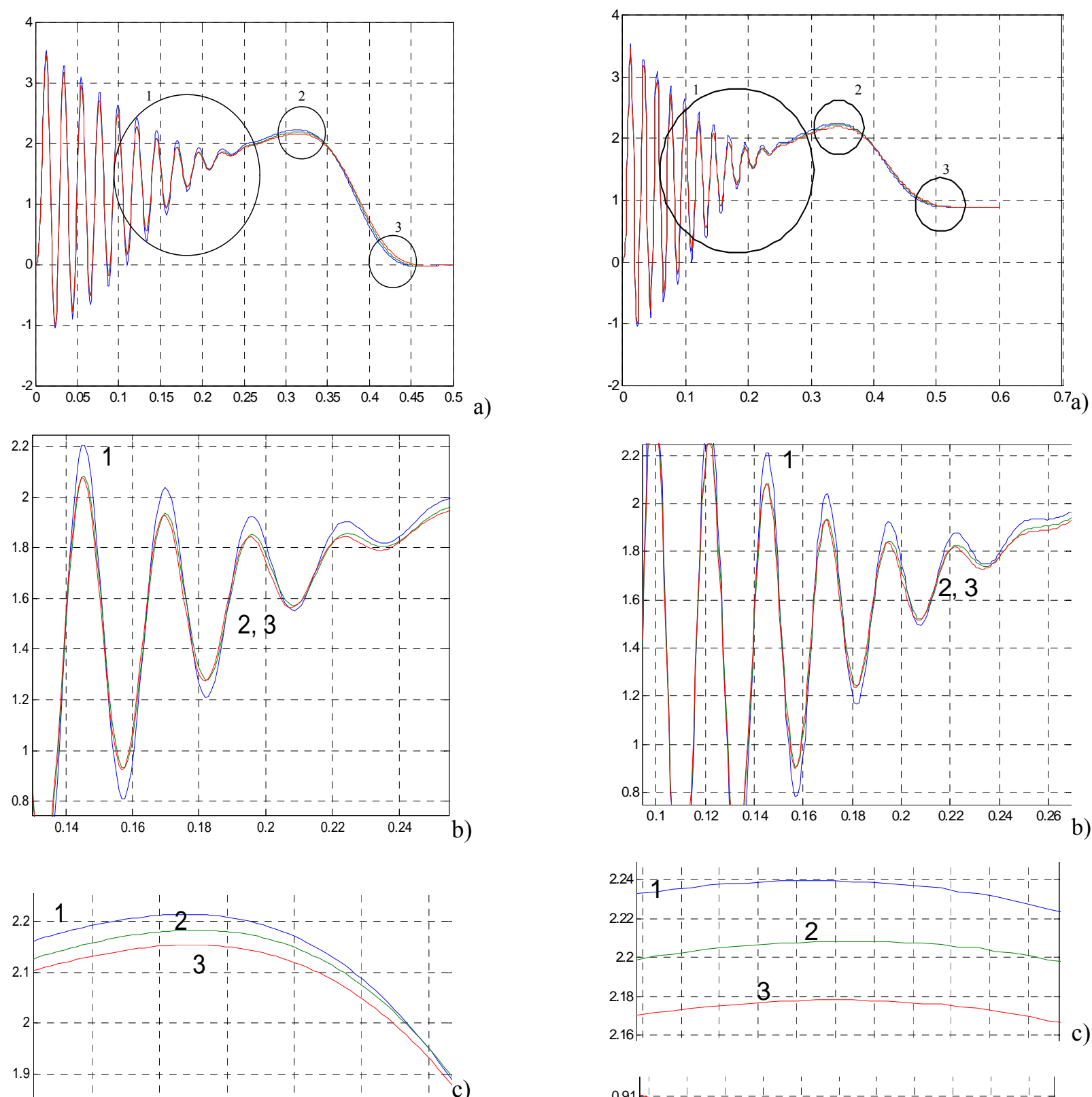

c)
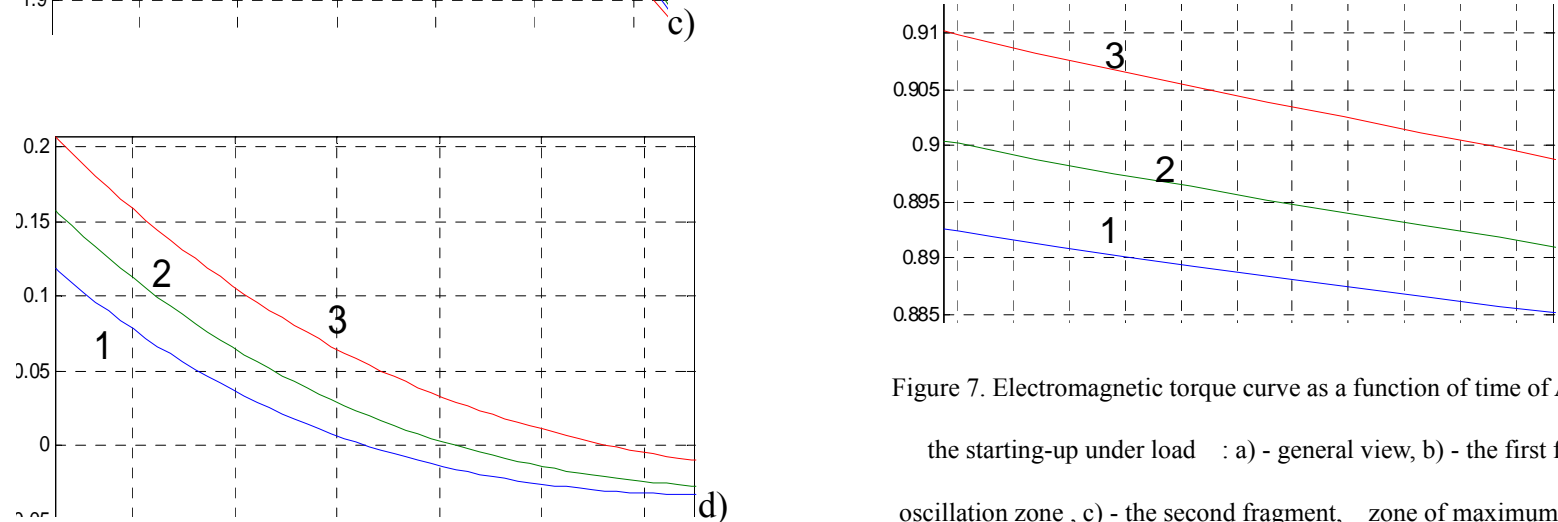

Figure 7. Electromagnetic torque curve as a function of time of AC Motor in the starting-up under load : a) - general view, b) - the first fragment, oscillation zone, c) - the second fragment, zone of maximum torque, d) -

Figure 6. Electromagnetic torque curve as a function of time of the third fragment, steady state zone. asynchronous motor under load at loadless starting : a) - general view, b) the first fragment, oscillation zone , c) - the second fragment, zone of maximum torque, d) - the third fragment, stationary regime zone. 

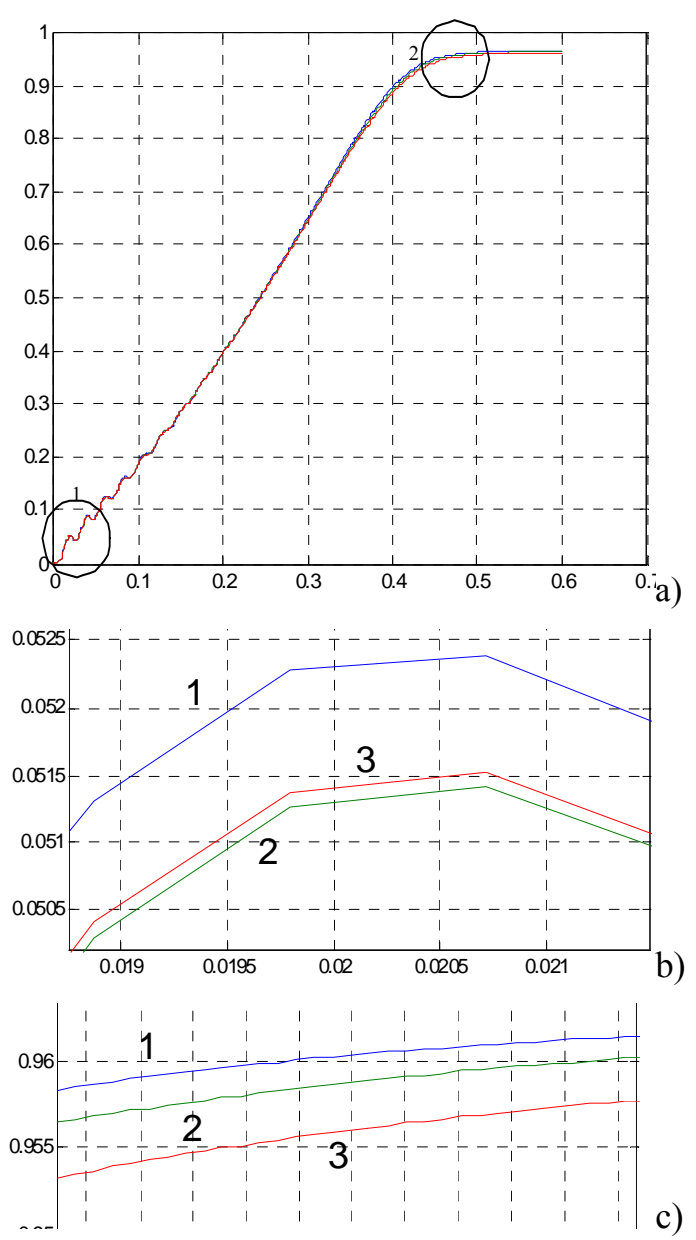

Figure 8. Speed rotation curve as a function of time of AC motor in the starting-up under load : a) - general view, b) - the first fragment, first oscillation , c) - second fragment, access to the stationary regime .

Table 2: Mismatch in modelling AC motor for various models

\begin{tabular}{|c|c|c|c|}
\hline \multicolumn{4}{|c|}{$\begin{array}{c}\text { calculation without load on the motor shaft } \\
\text { in the frequency of rotation }\end{array}$} \\
\hline \multicolumn{2}{|c|}{ in the torque } \\
\hline At starting & stationary regime & At starting & stationary regime \\
\hline $2 \%$ & $0,2 . .0,5 \%$ & $5,5 \%$ & $1 . .27 \%$ \\
\hline \multicolumn{4}{|c|}{ Calculation under load } \\
in the frequency of rotation & in the torque \\
\hline At starting & stationary regime & At starting & stationary regime \\
\hline $1,7 . .2,2 \%$ & $0,2 . .0,6 \%$ & $3,18 \%$ & $0,8 . .6,7 \%$ \\
\hline
\end{tabular}

The relative errors obtained, shown in Table 2, are small enough. In many cases, it would seem, such mismatches can be ignored. However, recent major studies have been devoted to developing algorithms for a vector drive which provides high precision control. In "the operation of control system keeping of speed rotor in zero as with nominal load and without, has been shown. The Error of maintaining of the zero speed is no more than $0,01 \mathrm{rad} / \mathrm{s} "[12$, p. 327 ]. Errors, which can be obtained in the simulation, considerably exceed the error control.

The real error may be substantially higher, since, in the simulation of electric drives, a real form of energy converter is not considered: voltage regulator (VR) or frequency converter (FC) (Fig. 9).
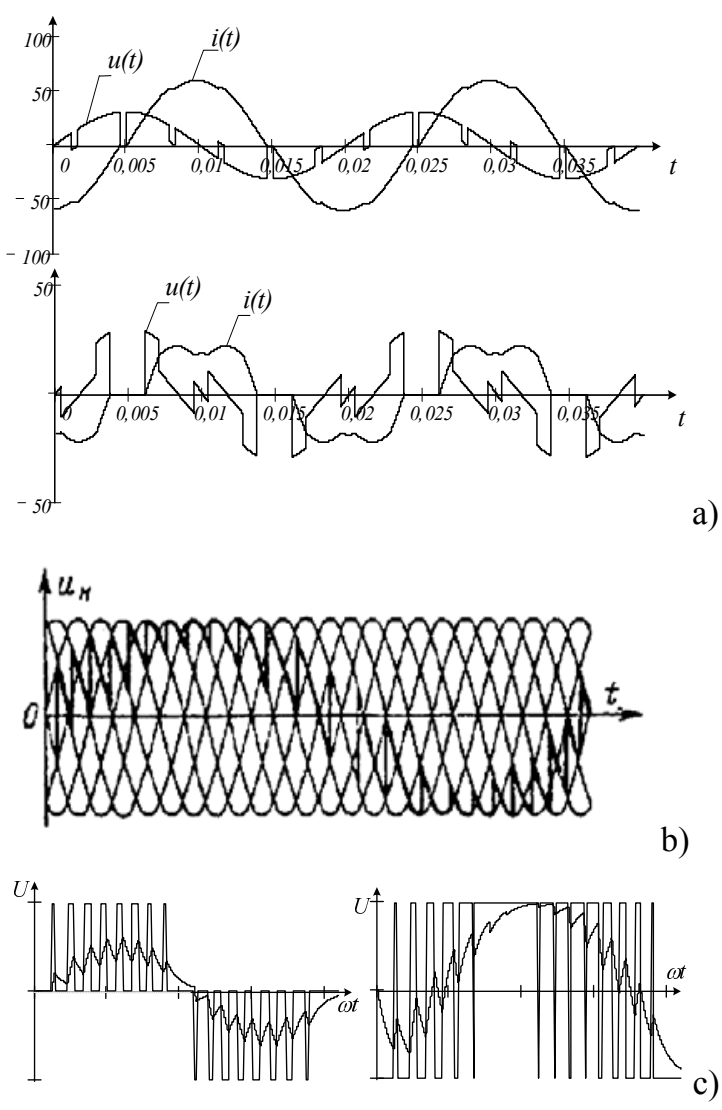

Figure 9. Electric drive current and voltage as a function of time: a) VR -

AC motor, b) NFC-AC motor, c) FC-AC motor with PWM control.

From the signal voltage power converters, we can see that all the signals have steep fronts during the commutation of the control switches. Value of the derivative voltage can make $20-500 \mathrm{~V} / \mathrm{ms}$. This means that there are discontinuities and, indeed, nonlinearity. Simulation of electrical drive with the real form of the 
supply voltage is a complex problem. At the same time the solution is almost impossible to verify, as for example, examples of electrical drive models from SimPower Systems Matlab libraries can be calculated only for those parameters specified in the settings for this model.

Through analysing the voltage of the system's autonomous inverter exit [frequency converter-asynchronous motor] (FC-Motor AC) (fig.9, c), it can be shown that, for certain values of the step of integration, information on the real voltage at the inverter exit will be lost. The narrow impulses of the tension at the beginning and end of a half-cycle are between the adjacent steps of an integration $h>\tau_{i}$ where $h$ integration step. The number of such voltage pulses depends upon modulation frequency $f_{m}$ and duty cycle $\gamma$. Figure 10 shows the variations in the duration of pulses $\tau$ depending on their numbers $i$ at the end of a half-period of the exit voltage. The curves in the same Figure correspond to modulation frequencies $4,8,16 \mathrm{kHz}$. Modulation frequencies are taken on the basis of studies used and applied in most industrial frequency converters. For comparison, we distinguish the curves obtained for an integration step of $1 \mu$ s (microsecond) (with such $h$, there is no information loss) and the limit mode is $50 \mu \mathrm{s}$.

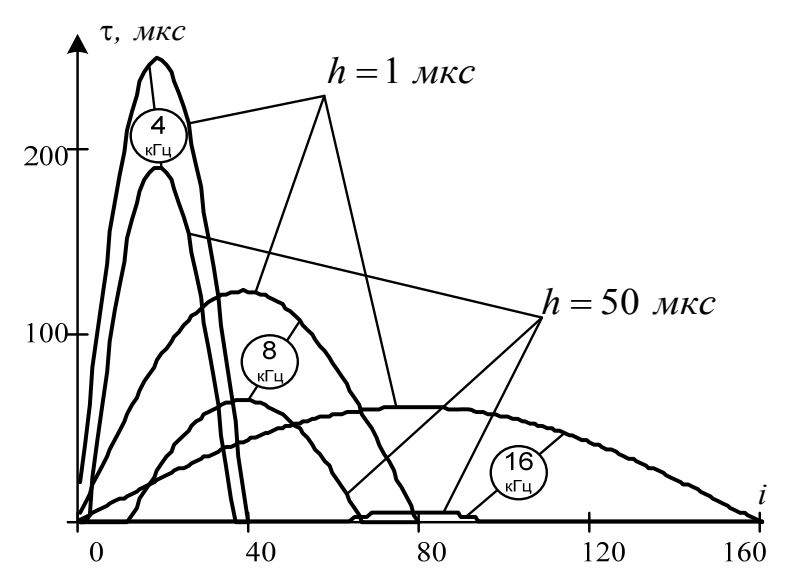

Fig. 10. variations in the duration of pulses $\tau$ depending on their number $i$ of a half-period of the output voltage

The results of the simulation of electrical drives run by frequency converters with a real form of the modulated voltage at the invertor exit show that a distortion of voltage occurs at the exit. For example, Figure 11 shows the results of simulation of output voltage for a frequency of $8 \mathrm{kHz}$ and a duty cycle $\gamma=$ 0.5 at the beginning of the initial alternation of the period in the $0 \ldots . .0 .0025 \mathrm{~s}$. interval. Voltage points calculated for integration steps of a) $10 \mu \mathrm{s}$ and b) $50 \mu \mathrm{s}$ are more marked on voltage pulses.
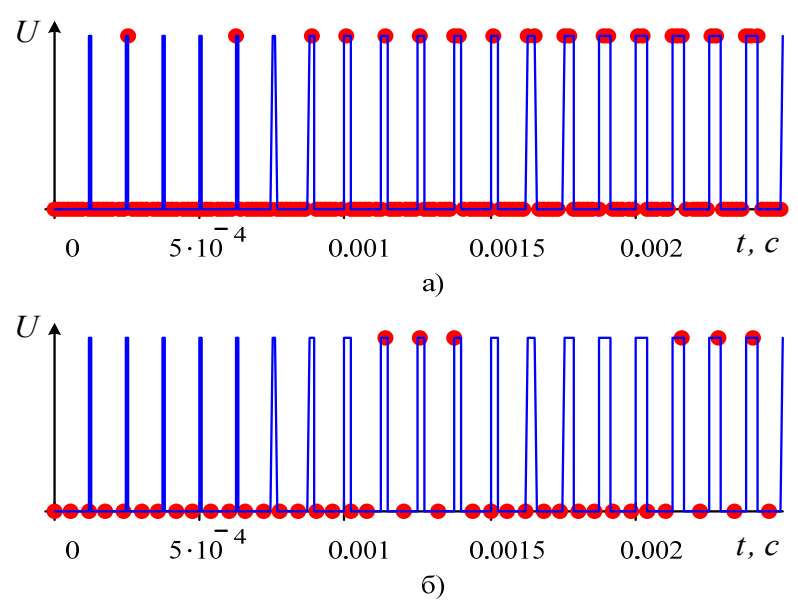

Figure 12. Simulation of voltage at the invertor exit with a modulation frequency of $8 \mathrm{kHz}$

In Figure 12, a reduction in bursts of pulses is clearly observable. This leads to errors during calculation of the voltage's fundamental harmonic.

Results of the study of discretisation error during the simulation are shown in Table 4. The study was carried out for evaluation of the effective value of the exit voltage of the autonomous invertor with modulation frequencies $f_{M}=4000 \mathrm{~Hz}, f_{M}=8000 \mathrm{~Hz}$ and an integration step $h=50 \mu$ s.

Relative error was calculated depending on exact value of voltage. As the results show (Table 4), the relative error of fundamental harmonic amplitude $\delta U_{\left(\frac{f}{f_{M}}\right)}$ depends on the coefficient of duty cycle $\gamma$ and is within the limits of $1 \ldots 17 \%$ for $f_{M}=4000 \mathrm{~Hz}$ and $1 \ldots . .27 \%$ for $f_{\mathcal{M}}=8000 \mathrm{~Hz}$.

Table 4. Evaluation of relative errors in simulation of the system's voltage (FC-Motor AC) with PWM 


\begin{tabular}{|c|c|c|c|}
\hline $\begin{array}{c}\text { Frequency PWM, } \\
f_{\mathcal{M}}, H z\end{array}$ & $\begin{array}{c}\text { Frequency } f \\
H z\end{array}$ & $\gamma$ & $\begin{array}{c}\text { Relative error of } \\
\text { voltage, } \%\end{array}$ \\
\hline \multirow{9}{*}{8000} & \multirow{3}{*}{50} & 1 & 0,692 \\
\hline & & 0,5 & 9,434 \\
\hline & & 0,25 & 11,079 \\
\hline & \multirow{3}{*}{25} & 1 & 1,968 \\
\hline & & 0,5 & 0,438 \\
\hline & & 0,25 & 21,548 \\
\hline & \multirow{3}{*}{10} & 1 & 0,486 \\
\hline & & 0,5 & 6,899 \\
\hline & & 0,25 & 17,312 \\
\hline \multirow{9}{*}{4000} & \multirow{3}{*}{50} & 1 & 0,82 \\
\hline & & 0,5 & 9,358 \\
\hline & & 0,25 & 27,241 \\
\hline & \multirow{3}{*}{25} & 1 & 0,229 \\
\hline & & 0,5 & 7,954 \\
\hline & & 0,25 & 8,637 \\
\hline & \multirow{3}{*}{10} & 1 & 0,149 \\
\hline & & 0,5 & 6,106 \\
\hline & & 0,25 & 18,397 \\
\hline
\end{tabular}

Figure 13 presents the curves of variation in average relative error for determination of the amplitude of the voltage depending on the frequency of the electrical drive system (FC-Motor AC) with PWM for $f_{M}=8000 \mathrm{~Hz}$ and $h=50 \mu \mathrm{s}$.

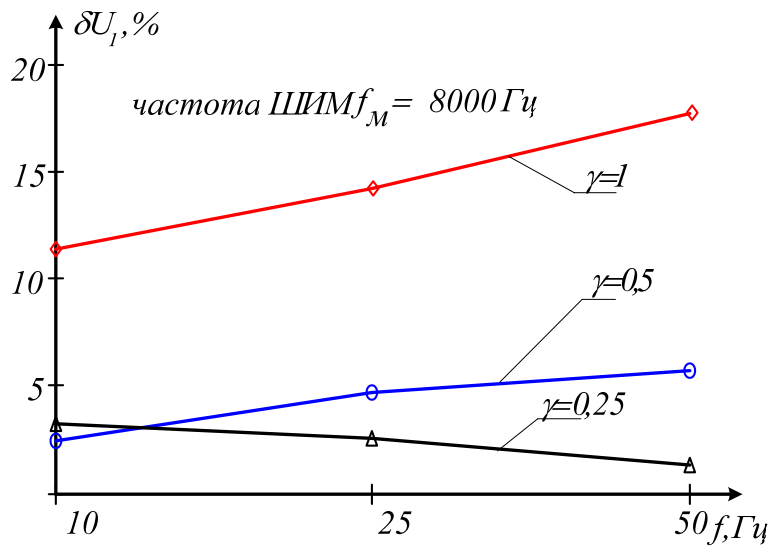

Fig.13. Functions of the average relative error for determination of voltage amplitude

Therefore, during simulation of asynchronous electrical drive systems run by invertors with voltage at PWM, we note the appearance of errors in determination of the voltage's integration characteristics. The reason is a form of signal following the presence of a large number of fronts caused by high modulation frequency $f_{M}=4000 \mathrm{~Hz}$ and $f_{M}=8000 \mathrm{~Hz}$. In this regard, the question comes up of choice of integration step, which should be chosen on the basis of calculation of modulation frequency $f_{M}$ and coefficient of duty cycle $\gamma$

The analysis carried out reveals the type of errors produced during simulation of alternative electrical drives, regulated asynchronous electrical drives in particular.

\section{Conclusions}

Increase in the precision of the methods employed in the resolution of the rigid differential equations systems (SDE) enables reduction of the percentage of error, but its value is located at the level $1.8 . .0 .5 \%$. Such data has been obtained only for the equation of order 2. For models of electromechanical systems constituted by 5 equations, error will be considerably higher.

The presence of nonlinearity, even for a two-mass system, gives not only quantitatively but qualitatively different solutions for different numerical methods. If there is no nonlinearity, all the numerical methods give the same result in the calculation. Simulation must precede the analysis of the frequency characteristics and the stability of the system. It is possible to have a preliminary computation to get an idea of the nature of the running processes and justification of the numerical method for integration of differential systems equations DSE depending on its structure for adequate quality solutions and low enough calculation errors.

For systems with discontinuous nonlinear functions, stable solutions can be obtained by methods with a constant step of integration and with preliminary 
evaluation of the step size.

In some cases, it appears that a more complete differential equation (when taking into account a large number of peculiarities of the system) gives worse results under inaccurate task conditions of initial data.

A more complete equation may be more sensitive to error variations.

Additional studies should be done on the asymmetry of motor parameters, as the system is not only nonlinear but also asymmetric, which will result in non-uniform assessment of accuracy of differential equations model resolution, as well as variables and nonlinear parameters.

\section{References}

[1] Karlaschuk V.I. Electronic lab to IBM PC. T. 1. Modeling elements of analog systems - 6 th ed. . M: Salon - P, 1999.- p. 590.

[2] German - Galkin S.G. Computer simulation of semiconductor systems in MATLAB 6.0 : Proc. manual for high schools. Edition - CROWN print, 2001.- p.320.

[3] Bacvalof N.C. Numerical method / Bakhvalov, N. Zhidkov, GM Kobelkov - 3rd ed., ext. and revised. - M.: BINOM Knowledge of Laboratory 2004. p.636

[4] E. Hairer, Nersett S., Wanner G. Solve ordinary differential equations. Nonrigid problems. Moscow: Mir, 1990, p. 512.

[5] E. Hairer, G. Wanner, Solve ordinary differential equations. Rigid and differential - algebraic problems. Moscow: Mir, 1999. p.685.

[6] Skvortsov L.M., Adaptive methods of numerical integration for modeling dynamic systems // Izv. RAN. Theory and control system. 1999. № 4. p. 72-78.

[7] Skvortsov L.M. Explicit adaptive methods for the numerical solution of stiff systems, mathematical modeling. T. 12. 2000. № 2 . p.97-107.

[8] Levenshtam V.B. Hatlamadzhiyan G.L. Distribution of homogenization theory to differential equations, containing a rapidly oscillating terms with large amplitudes. Problem of periodic solutions, "Izv. high schools. Math. 2006. № 6. p. $35-47$.

[9] Egorov A.I. Ordinary differential equations with applications / Yegorov - $22^{\text {nd }}$ ed. Corr. - Moscow: FIZMATLIT, 2005. p.384.

[10] Chornyi O.P. Peculiarities of numerical integration method of differential equations systems for electromechanical systems models. /Chornyi O.P., Berdai Abdelmajid // Electromechanical and energy-efficient systems. Kremenchug: National university of Kremenchug, 2010. - Vyp.3/2010 (11), p.46 -51. [11] Kopylov I.P. Mathematical modeling of electrical machines: Manual for high schools for spec. «Electrical machines". Moscow: High School, 1987, p.248s.

[12] E.M. Potapenko , Robust algorithms for vector control of asynchronous drive / EM Potapenko, Potapenko, EE. Zaporozhye . ZNTU, 2009, p.352.

[13] C.-M. Ong, Dynamic Simulation of Electric Machinery Using Matlab/Simulink, Upper Saddle River, Prentice Hall, 1998, 626p.

[14] Algorithm of Generating PWM converters for Modeling and Control of Electrical Drives. Chornyi O.P, O.V.Ostapenko, O.S. Vorobeichik, I.V. Urdìn, J.A. Topčiênko. // Electromechanical and energy-efficient systems. Kremenchug: National university of Kremenchug, 2010. Vyp.1/2009 (5), p.39 -42.

Berdai Abdelmajid. Was born in Rabat, Morocco in 1968. Now he is Assistant Professor in the National School of Electricity and Mechanics, ENSEM, University Hassan II Ain Chock Casablanca in Morocco. From 2002 to the present, he is a member of the Laboratory Building Technologies and Industrial Systems ( TCSI ), Research Group: Electrical Systems ( ESE ). Currently he is preparing his dissertation $(\mathrm{PhD})$ at the Automation control systems and electric drive department on speciality: Electrotchnical complexes and systems in Kremenchuk Mykhailo Ostrohradskyi National University. His research interests include Dynamic Simulation of Electric Machinery, Numerical integration methods of differential equations systems for electromechanical systems models, Simulation and optimisation of renewable energy systems. 
Abdelhadi El Moudden. Doctor of Science of The National Polytechnic Institute Of Toulouse (INPT) - FRANCE. $\mathrm{He}$ is now Professor habilité in the National School of Electricity and Mechanics, ENSEM University Hassan II Ain Chock, Casablanca, Morocco.

His research interests include Dynamic Simulation of Electric Machinery , Simulation and optimisation of renewable energy systems. He has presented and published many articles in scientific journals and conferences (IEEE).

Chornyi O.P.,Professor Doctor of Science in Kremenchuk Mykhailo Ostrohradskyi National State Polytechnic University. Ukraine. Now he is Director of the Institute of Electromechanics, energy saving and control systems. His research interests include the use of quality power conversion for monitoring of electromechanical equipment state, Estimation of modes and diagnosis of induction motors based on the quality of energy conversion, Dynamic Simulation of Electric Machinery. He has published numerous refereed papers in specialised journals and conferences. 